\title{
Participatory Learning Approach
}

\author{
Michael Bieber \\ New Jersey Institute of Technology, USA \\ Jia Shen \\ New Jersey Institute of Technology, USA \\ Dezhi Wu \\ New Jersey Institute of Technology, USA \\ Starr Roxanne Hiltz \\ New Jersey Institute of Technology, USA
}

\section{INTRODUCTION}

The Participatory Learning Approach (PLA, pronounced "play") engages students as active participants in the full life cycle of homework, projects, and examination. PLA's core idea is that students design the questions or projects, execute them, and then assess and grade their peers' solutions. Each stage can be performed by individuals or by teams. Students should be able to observe (read) everything their peers do so they can learn further from others' efforts.

Designing problems challenges students to critically assess understanding of a subject by their peers. This encourages students to analyze course materials in order to determine the most important aspects for this assessment. Evaluating solutions challenges students to assess how fully a set of materials (the solution) fits their understanding of the field as well as the problem posed.

PLA is designed to work for a wide range of students from junior high though graduate and professional schools, as well as for training and adult learning. PLA has the following major objectives:

- $\quad$ to increase learning of course materials (primarily) and assessment skills (secondarily); and

- to provide and evaluate a systematic, collaborative approach to homework assignments, projects, and examinations, focusing on active participation and peer evaluation;

\section{FOUNDATIONS}

PLA is grounded in constructivist theories of learning (Piaget, 1928; Vygotsky, 1978) which suggest that knowledge is actively constructed by, rather than transmitted to learners. People learn by applying their knowledge to meaningful problems (Brown, Collins \& Duguid, 1989; Hawkins \& Pea, 1987), actively building their own understanding.

Assessment and instruction typically are viewed as separate activities with different purposes. Some researchers have called for changing classroom culture so assessment becomes authentic-a fundamental part of the learning process (Shepard, 2000; Wright 2003). In PLA, assessment is closely tied to the learning process, in which students both assess other students' work and have their own work assessed.

Self-evaluation and peer evaluation contribute to learning in several ways. This awareness is facilitated when students are given specific criteria by which their own work is evaluated (Shephard, 2000). Students participating in assessment activities develop a metacognitive awareness of what constitutes excellent work (Frederickson \& Collins, 1989; Liu, Lin, Chiu \& Yuan, 2001). Students reported benefiting from needing to defend their opinions about their work (Klenowski, 1995) and from having access to their peers' work (McConnell, 1999). Knowing that their peers would read their assignments also motivated their learning (McConnell, 1999). 
An analysis of 62 studies showed that self-, peer, and co-assessment are effective tools for developing competencies required in professional organizations (Sluijsmans \& Moerkerke, 1999). Peer assessment practices should develop lifelong learning skills such as ability to evaluate one's own work and that of one's peers, which employers seek from students when they enter the professional world (Boud, 1990; Hargreaves, 1997).

\section{PLA PROCESS}

PLA embodies the following systematic process. The following description assumes that students work individually on each stage. Alternatively, collaborative groups could perform the task at any stage, which would further enhance learning.

Software supporting PLA could streamline the process for both students and instructors, reducing cognitive and administrative overload. Software to fully implement PLA is currently under development at the New Jersey Institute of Technology. In the meantime, classes could utilize learning management systems such as BlackBoard, WebBoard, and WebCT to post entries in a threaded manner (so that description, solution, and grades are grouped together for each problem). Instructors optionally may

Figure 1. Instructor and student processes within PLA. Solid arrows show the process flow. Dashed arrows emphasize that students also learn by observing everything their peers do.

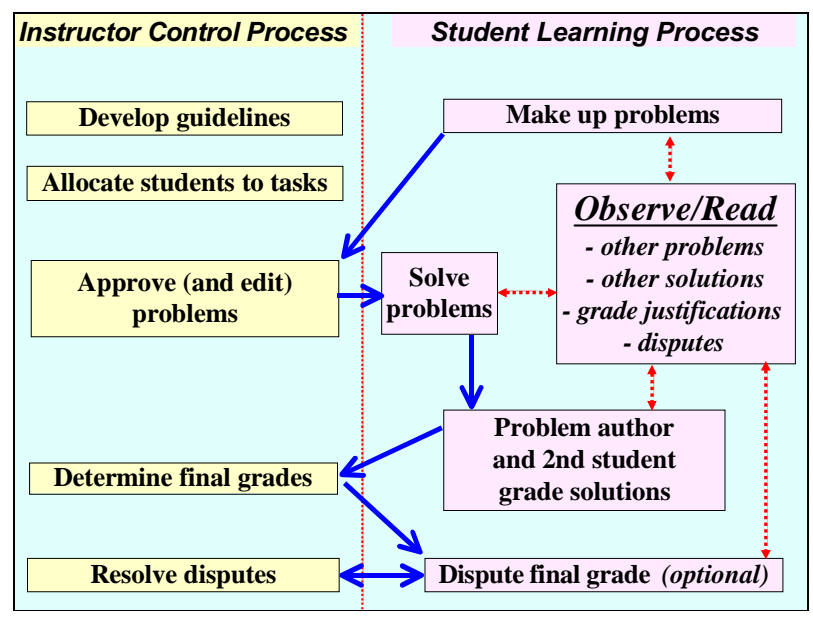

permit all entries to be posted anonymously, allocating an ID to each problem and telling students the ID of the problem they should work on.

The following description assumes the use of supportive software. Students should be able to read everything peers post online, which is an important learning component. Figure 1 illustrates the PLA process.

Software could assist with allocating students to PLA tasks (i.e., which problems to solve and grade). Software also would facilitate students observing their peers' activities. The stages of the PLA process include:

- $\quad$ Each student designs a problem, using guidelines provided by the instructor. Students post the problem description online.

- The instructor approves the problem description, editing it if necessary.

- $\quad$ Each problem is allocated to a different student who will solve it.

- $\quad$ Each student posts his or her solution online. Students grade the solution to the problem they authored, using guidelines provided by the instructor. Students may be required to grade using several different criteria. They must provide a written justification of at least two sentences for each evaluation criterion. Justifications, a detailed written critique-positive or negative-are a vital aspect of learning how to assess.

- Students are allocated a second solution to evaluate, providing each solution with a second opinion.

- Instructors assign a final grade to each solution, using the two student evaluations as input. If the two student grades are close enough (e.g., within 10 out of 100 points), to conserve time the instructor optionally may choose to accept the higher grade without re-evaluating the solution.

- Students optionally may dispute their grade, in which case they must re-grade their own solution using the same evaluation guidelines. Disputes are an especially important feature. They help ensure the fairness of the PLA approach, especially when instructors do not have time to carefully review each answer and evaluation. If a student believes the peer evaluations were 
incorrect, a dispute will ensure that the instructor focuses adequate attention to this specific problem.

- The instructor determines the final grade for the disputed solution.

\section{Opportunities for Learning}

PLA incorporates several opportunities for learning, in designing problems, answering them, grading them, disputing results, and in observing/reading what everyone else does.

PLA forces students to view a subject from several new vantage points. Designing problems challenges students to analyze course materials in order to determine the most important aspects for critically assessing understanding of a subject by their peers. Evaluating solutions and arbitrating disputes challenge students to assess how fully a set of materials (the solution) fits their understanding of the field as well as the problem posed. Often, solutions will be quite different from how the evaluator views the problem, forcing a rethinking of the material. The PLA stages of problem design, solution design, solution evaluation, and dispute arbitration take place over a period of time, and often the class will have moved on to new materials while the latter stages are being conducted. This repetition provides reinforcement. The extended time span fosters synthesis, as students revisit prior topics at the same time they are learning new materials.

Finally, students learn from reading what their peers have written — problems, solutions, grading justifications, and disputes. Students read their peers' work out of curiosity, for new ideas, and for comparison to their own work.

\section{Extending the Basic PLA Process}

PLA can be extended in several ways from this basic model.

- Collaboration: Groups of students could work together to create problems, solve problems, evaluate, arbitrate disputes, and assign final grades.

- Multiple Problems: Students could design and solve more than one problem within a single assignment (homework, project, quiz, or exam), and thus be involved in various roles for different problems under the same assignment.

- Sub-Problems: Problems could be designed to contain several sub-problems. Each could be conducted in tandem or sequentially when the prior part was complete. Each sub-problem would be assessed separately. For sequential sub-problems, students would not start solving the next sub-problem until the prior one passed assessment.

An example of two sequential sub-problems could be: i) the design of a large computer system, followed by ii) its implementation and testing. The instructor may want the class to use PLA to assess the design before students move on to the implementation. Similarly, the instructor may break a semester project into an extended outline and its completion, and use PLA to evaluate the outline before students complete it.

For complex problems, the individual or group designing the problem may wait with designing a subsequent sub-problem until its prior subproblem has been completed.

- Multiple Evaluation Opinions: The PLA model supports any number of evaluations for each problem, instead of always two.

- Separating Evaluation from Grading: For larger projects and in cases where instructors may wish to limit potential bias, PLA will support separating the assessment of the solution from its grade. One evaluator would write up a detailed evaluation. A second evaluator would assign a grade based on the evaluation. The instructor should separate evaluation instructions from grading instructions.

- Evaluating Other PLA Activities: In addition to evaluating solutions, the PLA model supports evaluating other PLA activities, such as grading problem statements, dispute arbitration, and even other evaluations, and when separated from the other evaluations, grades. This could motivate students to take these other aspects more seriously and learn more deeply from them. Such additional evaluations could also be disputed and arbitrated.

- Activity Rejection: Evaluation could be used to reject any activity (problem statement, subproblem solution, etc.) that is so poor that the 
process cannot continue effectively with it. In this case the activity could be redone and reevaluated, or the evaluator or instructor would provide a substitute, and the PLA process would continue. For example, when evaluating a semester project outline (a solution to the first semester project sub-problem), the evaluators could determine it not strong enough and reject it. In this case the group that created the semester project outline would have to redo it, hopefully benefiting from the evaluation justification.

\section{EXPERIENCES AND ISSUES}

Many interesting issues arise when embedding peer assessment within learning activities. Pragmatic concerns also arise when coordinating a new teaching approach. The observations that follow are based upon several years of using PLA in online participatory exams (Shen, Hiltz, Cheng, Cho \& Bieber, 2001; Shen, Cheng, Bieber \& Hiltz, 2004; Wu, Bieber, Hiltz \& Han, 2004), as well as the reported empirical results of other researchers who have employed aspects of the PLA approach.

Thus far, in our empirical studies of the participatory examination, students report that they learn from all phases of the process (e.g., making up questions and grading answers, as well as studying for and answering their questions); that the participatory exam process is more enjoyable and, overall, more satisfying than the traditional exam process; but that they have some questions about the fairness of being graded by peers.

Students have concerns when working with PLA. They often believe it is the instructor's job to evaluate students. They feel unqualified to evaluate their peers, and indeed they often have no practice within an academic setting. Furthermore, when everyone in the class is responsible for evaluating a few questions, quality can be quite inconsistent. For all these reasons, there sometimes is a perception that PLA is inherently unfair. Therefore it is critically important to explain to students that they indeed are qualified to assess concepts and people, and that they will often do this in the workplace (Hersam, Luna \& Light, 2004).
Equally important is assessment training and repeated experience (Kerr \& Park 1995). Assessment is a skill that must be learned. Training, experience, and excellent guidelines should improve inconsistency in grading, and the ability to grade in groups should also help. One can also build assessment training into the PLA support software.

Instructors can incorporate inquiry-based methods within PLA to better guide students in developing problems, solutions, and evaluations. For example, this could lead students in appropriate ways to find and analyze information when solving specific kinds of problems.

Instructors can involve students in planning assignments. Several possible activities could get students to start thinking about developing problem statements, including developing a common concept map of the domain, creative brainstorming, and so forth. Students also could be involved in designing the assessment criteria (Hersam et al., 2004).

Students often are concerned about the lack of privacy when the process is not anonymous. Pressure to award positive evaluations to peers is offset by the desire for integrity when evaluations are entirely visible. Anonymity most likely is not practical in smaller classes when multiple sections cannot be combined for PLA activities.

Cheating is always an issue with online systems. Instructors should take advantage of anti-plagiarism services that are becoming available. Answers cannot generally be copied from classmates because each student (group) makes up different problems. However, the danger always persists that students will find someone else to do their work.

A practical issue concerns the amount of time the PLA takes. When instructors make up questions and then grade, students generally do the assignment and receive results quickly. The PLA process can take up to three weeks for classes meeting once a week and where part-time students might only allocate one day a week to homework. Instructors may need to engineer their classes so multiple PLA processes at different stages are underway at the same time (grading, answering questions, formulating new problems-each from a different topic in the course). This actually builds in opportunities for reinforcement and synthesis as students work on materials from several topics at the same time. 
A fascinating challenge for enhancing both quality and fairness will be to structure ways for students to dispute a grade if they feel it is too high. Doing so would be instructive for the disputing student, who would have to justify why his or her work was overrated, for the evaluator who potentially overrated, and the dispute arbitrator. One approach would be to award bonus points greater than the grade were the dispute successful. The graders could optionally lose points for grading poorly.

The right to dispute evaluations acts as a safety net for many fairness issues. Disputes can rectify most cases where students feel something has gone wrong, and instructors should take them seriously. The PLA model supports involving students in collaborative dispute arbitration, adding another level of learning to the process.

\section{SUMMARY}

PLA embodies a student-centered learning approach that trains students to assess and builds the selfassurance to assess confidently. Such evaluation is a valuable, lifelong, everyday skill that incorporates critical thinking and analysis. In the classroom, the active participation and collaboration of the PLA process results in deeper learning as well as increased awareness of, and interest in, the issues surrounding topics covered in class. PLA's success comes from the increased control students have over the process, the flexibility in the process (that it takes place over a period of time), and that students learn in so many ways. PLA fosters more knowledgeable students, confident and skilled in collaboration and subject-, self-, and peer-assessment.

\section{REFERENCES}

Boud, D. (1990). Assessment and the promotion of academic values. Studies in Higher Education, 15(1), 101-111.

Brown, J.S., Collins, A. \& Duguid, P. (1989). Situated cognition and the culture of learning. Educational Researcher, 18(1), 32-42.

Frederickson, J.R. \& Collins, A. (1989). A systems approach to educational testing. Educational Researcher, 18, 27-32.
Hargreaves, D.J. (1997). Student learning and assessment are inextricably linked. European Journal of Engineering Education, 22(4), 401-410.

Hawkins, J. \& Pea, R. (1987). Tools for bridging everyday and scientific thinking. Journal for Research in Science Teaching, 24(4), 291-307.

Hersam, M., Luna, M. \& Light, G. (2004). Implementation of interdisciplinary group learning and peer assessment in a nanotechnology engineering course. Journal of Engineering Education, 93, 49-57.

Kerr, P. \& Park, K. (1995). Peer grading of essays in a Principles of Microeconomics course. Journal of Education for Business, 70(6), 357-362.

Klenowski, V. (1995). Student self-evaluation process in student-centered teaching and learning contexts of Australia and England. Assessment in Education, 2, 145-163.

Liu, E., Lin, S., Chiu, C. \& Yuan, S. (2001). Webbased peer review: The learner as both adapter and reviewer. IEEE Transactions on Education, 44(3), 246-251.

McConnell, D. (1999). Examining a collaborative assessment process in networked lifelong learning. Journal of Computer Assisted Learning, 15(3), 232-243.

Piaget, J. (1928). Judgment and reasoning in the child. London: Routledge \& Kegan Paul.

Shepard, L.A. (2000). The role of assessment in a learning culture. Educational Researcher, 19(7), 4-14.

Shen, J., Cheng, K.E., Bieber, M. \& Hiltz, S.R. (2004). Traditional in-class examination vs. collaborative online examination in asynchronous learning networks: Field evaluation results. Proceedings of the Americas Conference on Information Systems, New York.

Shen, J., Hiltz, S.R., Cheng, K., Cho, Y. \& Bieber, M. (2001). Collaborative examinations for asynchronous learning networks: Evaluation results. Proceedings of the 34th Hawaii International Conference on Systems Sciences. Los Alamitos, CA: IEEE Computer Society Press. 
Sluijsmans, D.M.A. \& Moerkerke, G. (1999). Creating a learning environment by using self-, peer-, and co-assessment. Learning Environments Research, 1, 293-319.

Wu, D., Bieber, M., Hiltz, S.R. \& Han, J. (2004). Constructivist learning with participatory examinations. Proceedings of the 37th Hawaii International Conference on Systems Sciences. Los Alamitos, CA: IEEE Computer Society Press.

Vygotsky, L. (1978). Mind in society: The development of higher psychological processes. Cambridge, MA: Harvard University Press.

\section{KEY TERMS}

Assessment: Determining how much someone has learned or how well someone understands a particular subject.
Authentic Learning: Learning that uses realworld problems and projects, and that allows students to explore and discuss these problems in ways that are relevant to them.

Constructivism: A theory of learning and knowing that holds that learning is an active process of knowledge construction in which learners build on prior knowledge and experience to shape meaning and construct new knowledge.

Participatory Learning Approach: A pedagogical approach involving students in the entire learning process involving a problem, including authoring, solving, and evaluating a problem and its solution.

Peer-Evaluation: Where peers (such as students) evaluate each others' solutions to problems or other assignments.

Self-Evaluation: Where a person evaluates his or her own solution to a problem or other assignment. 